\title{
Bacteremia Is a Risk Factor for Cerebrospinal Fluid Infection in Patients with Cerebrospinal Fluid Drains-A Retrospective Study
}

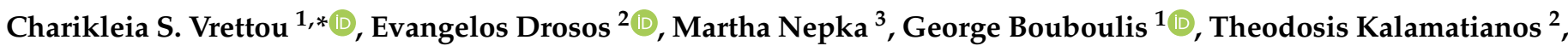 \\ Christina Liakopoulou ${ }^{1}$, Grigorios Gkouvelos ${ }^{1}$, Anastasia Kotanidou ${ }^{1}{ }^{1}$ and George Stranjalis ${ }^{2}$ \\ 1 First Department of Critical Care Medicine \& Pulmonary Services, Medical School of National and \\ Kapodistrian University of Athens, Evangelismos Hospital, 10676 Athens, Greece; \\ gbouboulis@gmail.com (G.B.); chr1stieliak@gmail.com (C.L.); guvelosgr@gmail.com (G.G.); \\ akotanid@gmail.com (A.K.) \\ 2 Department of Neurosurgery, Evangelismos Hospital, National and Kapodistrian University of Athens, \\ 10676 Athens, Greece; drosose@gmail.com (E.D.); tkalamatian@med.uoa.gr (T.K.); \\ gstranjalis@med.uoa.gr (G.S.) \\ 3 Department of Microbiology, Evangelismos Hospital, 10676 Athens, Greece; \\ microbiologiko@evaggelismos-hosp.gr \\ * Correspondence: vrettou@hotmail.com
}

Citation: Vrettou, C.S.; Drosos, E.; Nepka, M.; Bouboulis, G.;

Kalamatianos, T.; Liakopoulou, C.; Gkouvelos, G.; Kotanidou, A.; Stranjalis, G. Bacteremia Is a Risk Factor for Cerebrospinal Fluid Infection in Patients with Cerebrospinal Fluid Drains-A Retrospective Study. Bacteria 2022, 1, 48-55. https://doi.org/10.3390/ bacteria1010005

Academic Editor: Bart C. Weimer

Received: 26 December 2021

Accepted: 2 February 2022

Published: 8 February 2022

Publisher's Note: MDPI stays neutral with regard to jurisdictional claims in published maps and institutional affiliations.

Copyright: (C) 2022 by the authors. Licensee MDPI, Basel, Switzerland. This article is an open access article distributed under the terms and conditions of the Creative Commons Attribution (CC BY) license (https:// creativecommons.org/licenses/by/ $4.0 /)$.

\begin{abstract}
There is little evidence on the role of prior infection in patients with external ventricular drains (EVDs) and lumbar drains (LDs). In this study, our aim is to investigate whether previous bacteremia is a risk factor for cerebrospinal fluid drain infection (CSFDI) in patients with EVDs and LDs and to describe the microorganisms implicated. We designed a retrospective, single-center cohort study. We recorded patients' demographic and clinical characteristics, as well as microbiology laboratory data. We used non-parametric statistical methods to identify possible risk factors for CSFDI. We found 799 neurosurgical admissions during the study period, 70 of which fulfilled the inclusion criteria. Acinetobacter baumannii was the most frequent single pathogen isolated in the cerebrospinal fluid (CSF). Acinetobacter baumannii bacteremia was more common in patients with Acinetobacter baumannii CSFDI ( $p=0.01$ ). The distribution of the pathogens in the CSF differed from that of the pathogens isolated in blood $(p=0.001)$. In the univariate analysis, prior bacteremia was more common in patients with CSFDI ( $p=0.027)$, but, in the multivariate model, prior bacteremia was not identified as an independent risk factor ( $\mathrm{OR}=0.456, \mathrm{CI}: 0.138-1.512, p=0.2)$. In an ICU population, the most frequently isolated pathogens were Gram-negative Enterobacteriaceae and Acinetobacter baumannii. Previous bacteremia was significantly more probable among patients with EVDs or LDs who developed a CSFDI, and its role warrants further investigation.
\end{abstract}

Keywords: external ventricular drainage (EVD); lumbar drainage (LD); Acinetobacter baumannii; cerebrospinal fluid infection

\section{Introduction}

Severely ill patients with neurological injury and cerebrospinal fluid (CSF) drains often need treatment in an intensive care unit (ICU), where their survival probability can increase. At the same time, these patients are at risk of developing ICU-related complications and, in particular, ICU-acquired infections. The high risk of acquiring an infection in the ICU environment is attributed to invasive procedures that require breaking the skin barrier, e.g., vascular catheterization, to the use of orotracheal airways for providing mechanical ventilation, and to the deranged immunity that may accompany critical illness $[1,2]$.

Many studies have investigated the risk factors for cerebrospinal fluid drain infection (CSFDI) and the effectiveness of preventive strategies. Fewer focus on critically ill patients. The role of previous infection in the pathogenesis of CSFDI has, to our knowledge, only been addressed in one study, where prior chest infection was found to be a risk factor for 
ventriculitis [3]. The role of bacteremia in patients with cerebrospinal fluid drains (CSFDs) has not been examined, even though bacteremia is a known risk factor for CSF infection in patients with ventriculoatrial (VA) shunts. In this retrospective study, we, therefore, aim to investigate the possible role of previous, hospital-acquired bacteremia as a risk factor for CSFDI in a critically ill population with external cerebrospinal fluid drains, i.e., either external ventricular drains (EVDs) or lumbar drains (LDs).

\section{Results}

We reviewed all ICU admissions from 1 January 2011 to 31 December 2020, and we identified 799 neurosurgical cases. Figure 1 shows the study flowchart. A total of 70 patients were included in the final analysis. Table 1 shows the demographic and clinical characteristics of included patients. All EVDs were placed in the operating room while all LDs were placed at the bedside. A CSFDI was diagnosed in 28 out of the 70 patients (40\%). Patients with CSFDIs, compared with those without a CSFDI, had a higher cerebrospinal fluid (CSF) cell count (320 (53.5-1235) vs. 12.5 (5.25-90.75), $p<0.001)$, lower CSF glucose (51 (9-67) vs. 66.5 (52-97.5), $p=0.006)$, and higher CSF protein (93 (89-255) vs. 67 (34-140), $p=0.002)$.

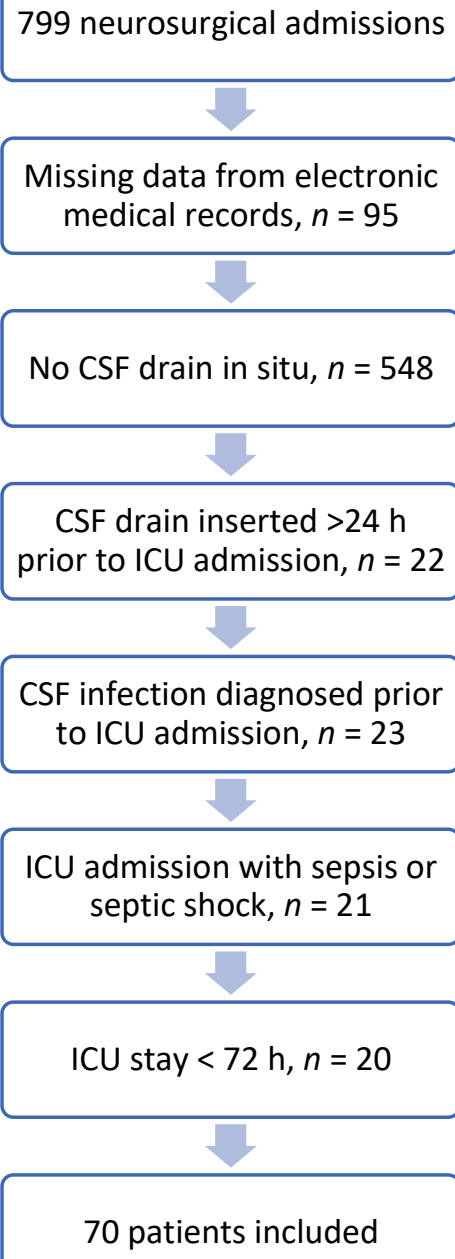

Figure 1. Flow chart of the patients. A total of 70 patients older than 18 years old fulfilled the inclusion criteria from January 2011 to December 2020. Horizontal arrows indicate the reasons for exclusion. CSF: cerebrospinal fluid; ICU: intensive care unit. 
Table 1. Demographic and clinical characteristics of patients with CSF foreign bodies and univariate analysis.

\begin{tabular}{|c|c|c|c|}
\hline & $\begin{array}{l}\text { CSF Infection Present } \\
\qquad n=28\end{array}$ & $\begin{array}{l}\text { CSF Infection Absent } \\
\qquad n=42\end{array}$ & $p$-Value \\
\hline Age $^{1}$ & $56(44.75-70)$ & $52.5(45.5-61.25)$ & 0.890 \\
\hline $\operatorname{Sex}^{2}$ & & & 1.000 \\
\hline Male & 16 & 23 & \\
\hline Female & 12 & 19 & \\
\hline Reason for ICU admission ${ }^{2}$ & & & 0.985 \\
\hline Ischemic Stroke & 1 & 2 & \\
\hline Intracranial Hemorrhage & 11 & 15 & \\
\hline Subarachnoid Hemorrhage & 7 & 12 & \\
\hline Traumatic Brain Injury & 3 & 3 & \\
\hline Brain Tumor & 6 & 9 & \\
\hline Foreign body type $^{2}$ & & & 0.349 \\
\hline EVD & 21 & 36 & \\
\hline LD & 7 & 6 & \\
\hline $\begin{array}{l}\text { Antibiomicrobial-impregnated } \\
\text { catheters used }^{2}\end{array}$ & & & 0.352 \\
\hline Yes & 3 & 10 & \\
\hline No & 20 & 32 & \\
\hline $\begin{array}{c}\text { Previously cultured pathogens in blood } \\
\text { cultures }{ }^{2}\end{array}$ & & & 0.027 \\
\hline Yes & 20 & 18 & \\
\hline No & 8 & 24 & \\
\hline $\begin{array}{l}\text { Number of CSF samples sent before } \\
\text { bacterial growth }{ }^{1}\end{array}$ & $3(2-5)$ & $3.5(2-5)$ & 0.473 \\
\hline Foreign body days ${ }^{1}$ & $14(10.75-18)$ & $12(7-18.25)$ & 0.214 \\
\hline Length of ICU stay ${ }^{1}$ & $31.5(23.75-52)$ & $25.5(9-33.25)$ & 0.037 \\
\hline ICU outcome $^{2}$ & & & 0.039 \\
\hline Survival & 14 & 32 & \\
\hline Death & 14 & 10 & \\
\hline
\end{tabular}

${ }^{1}$ Median (interquartile range)-Mann-Whitney $U$ test; ${ }^{2}$ Number of cases-Fisher exact test; CSF: cerebrospinal fluid; ICU: intensive care unit; EVD: external ventricular drain; LD: lumbar drain.

Figure 2 shows the relative frequencies of different pathogens isolated in the CSF alongside the corresponding relative frequencies of pathogens isolated in blood. We found that the distribution of the pathogens differed significantly in the two sample types $(p=0.0010)$. A post hoc analysis indicated that this difference may be attributed to the higher percentage of Staphylococcus epidermidis and of other Enterobacteriaceae in the blood and to the higher percentage of Acinetobacter baumannii in the CSF. Other Enterobacteriaceae identified but not individually shown in Figure 2 include Stenotrophomonas maltophillia, Proteus mirabilis, Escherichia coli, Haemophilus influenzae, Enterobacter cloacae, and Serratia marcescens. Other Gram-positive microorganisms identified but not individually shown include Enterococcus faecium, Staphylococcus hominis, Staphylococcus capitis, and Streptococcus haemolyticus. The difference in the relative frequencies of pathogens persisted even when the cases of Staphylococcus epidermidis were excluded from the analysis $(p=0.0212)$. Fisher's exact test, comparing the relative frequency of Acinetobacter baumannii CSFDI occurrence between patients with and without Acinetobacter baumannii bacteremia, showed that Acinetobacter baumannii CSFDI was more common in the former $(p=0.01)$. Pathogens identified in the CSF of patients with EVDs were Acinetobacter baumannii $n=8$, 
Klebsiella pneumoniae $n=6$, Pseudomonas aeruginosa $n=3$, Gram-positive cocci $n=2$, Candida spp. $n=1$, and Providencia stuartii $n=1$. Pathogens identified in the CSF of patients with LDs were Acinetobacter baumannii $n=3$, Pseudomonas aeruginosa $n=1$, Gram-positive $\operatorname{cocci} n=2$, and Candida spp. $n=1$. There was no statistical significance in the distribution of bacteria between cases with EVDs and LDs (Fisher's exact test, $p=0.284$ ).

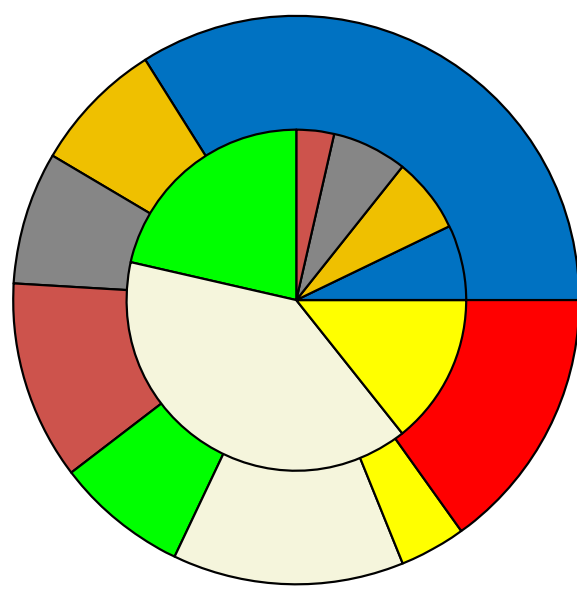

\begin{tabular}{|l}
$\square$ Staphylococcus epidermidis \\
$\square$ Other Gram+ microorganisms \\
$\square$ Candida species \\
$\square$ Providencia stuartii \\
$\square \quad$ Klebsiella pneumoniae \\
$\square$ Acinetobacter baumannii \\
$\square$ Pseudomonas aeruginosa \\
$\square$ Other Enterobacteriaceae \\
\hline
\end{tabular}

Figure 2. Comparative pie chart of the relative frequencies of the most frequently identified pathogens. CSF (inner disc) vs. blood (annulus); CSF isolates: Acinetobacter baumannii $n=11(39.3 \%)$, Klebsiella pneumoniae $n=6(21.4 \%)$, Pseudomonas aeruginosa $n=4(14.3 \%)$, Staphylococus epidermidis, and other Gram-positive pathogens, including Enterococcus faecium, Staphylococcus hominis, Staphylococcus capitis, and Streptococcus haemolyticus $n=4(14.2 \%)$, Candida spp. $n=2(7.1 \%)$, and Providencia stuartii $n=1$ (3.6\%). Blood isolates: Staphylococus epidermidis $n=18(34 \%)$, Acinetobacter baumannii $n=8(15.1 \%)$, other Enterobacteriaceae, including Stenotrophomonas maltophilia, Proteus mirabilis, Escherichia coli, Haemophilus influenzae, Enterobacter cloacae, and Serratia marcescens $n=8(15.1 \%)$, Providencia stuartii $n=6(11.3 \%)$, Klebsiella pneumoniae $n=4(7.5 \%)$, Candida spp. $n=4(7.5 \%)$, other Gram-positive pathogens $n=4(7.5 \%)$, and Pseudomonas aeruginosa $n=2(3.8 \%)$.

The presence of bacteremia was the only variable that differed significantly between the groups of patients with or without a positive CSF culture. Bacteremia was, therefore, the only risk factor identified by the univariate analysis. However, when we performed a logistic regression, including the presence of bacteremia, the type of CSFD, the length of stay, possible impregnation with antibiotics, and the number of CSF samples sent, bacteremia was not found to be an independent risk factor for CSFDI (OR = 0.456, CI: 0.138-1.512, $p=0.2)$.

\section{Discussion}

Recent literature on the pathophysiology of CSFDI is supportive of a retrograde route of bacterial invasion from the distal portion of the catheter lumen to the proximal portion in the ventricular system or, alternatively, of an inoculation of pathogens at the time of drain insertion $[1,4,5]$. A hematogenous spread to the CSF has been described for VA shunts but not for EVDs [1]. Moreover, very little is known regarding the significance of previous infection and bacterial colonization in patients who are at risk of developing a CSFDI [6-10]. In one study [8], where risk factors for CSFDIs were investigated, concomitant extracranial infection was found to be a statistically significant risk factor in critically ill patients with EVDs. The role of bacteremia has also been addressed in two more studies that included small numbers of neonates who developed a CSFDI $[9,10]$. To the best of our knowledge, there are no studies that address bacteremia as a risk factor for CSFDI in critically ill adults, even though this population is widely accepted as particularly vulnerable [6-8].

The risk factors for CSFDI mostly discussed in the literature are the duration of catheterization, the placement technique, the use of antimicrobial-impregnated catheters, the frequency of catheter manipulation and sampling, the presence of blood in the CSF, the 
type of cleansing and dressing, and the leakage of CSF [2,11-14]. From this list, we included in our analysis the factors that we could safely document in retrospect, namely, the use of antimicrobial-impregnated catheters, the number of CSF samples sent, and the duration of catheterization. None of them was found to be statistically significant in our population. This can be attributed to the relatively small number of patients finally included in our analysis and to the limited use of lumbar and antimicrobial-impregnated drains in our department. The role of CSF sampling in the emergence of CSFDIs is controversial [2,15-17], with some authors even advocating regular CSF sampling to detect a possible infection early. Our analysis showed that, in contrast to these factors, the presence of bacteremia was significantly more frequent in patients who eventually had a positive CSF culture. Even though it was not established as an independent risk factor in the multivariate model, bacteremia was the only variable that differed significantly between patients with and without CSFDI. This association could be explained by considering either the bloodstream mechanism or the patient's condition. Bloodstream spread may have a more prominent role in the occurrence of ventriculitis than previously recognized, possibly because the systemic inflammatory response, often associated with systemic infection, modifies the blood-brain barrier permeability, thus, facilitating microbial penetration into the central nervous system. On the other hand, it is possible that the same factors that render the patient susceptible to extracranial infection, such as a long duration of stay or a high illness severity score, also increase the risk of intracranial infection.

Historically, most CSFDIs have been attributed to Gram-positive pathogens and to the head skin flora but, lately, Acinetobacter baumannii and Gram-negative Enterobacteriaceae are increasingly implicated $[7,8,14,18,19]$, and this can be explained, at least partly, by their increasing levels of resistance to antibiotics and, in particular, to carbapenems [7]. In our sample, Acinetobacter baumannii was the most frequent pathogen and, together with Klebsiella Pneumoniae and Pseudomonas aeruginosa, comprised approximately $70 \%$ of ventriculitis cases. This could explain why Acinetobacter baumannii CSFDI was more common in patients with prior Acinetobacter baumannii bacteremia. On the other hand, some groups of pathogens were found in blood cultures but not in the CSF, the most prominent representative being the "other Enterobacteriaceae" group. Acinetobacter spp. is often transmitted to patients via persistence on environmental surfaces and transient colonization of the hands of health care workers [20]. It is hypothesized that Acinetobacter baumannii persists in medical environments, resists antimicrobials, and causes disease because of its capacity to form biofilms on solid surfaces [3]. Inappropriate empirical antibiotic therapy, comorbidities, neutropenia, and the presence of disseminated intravascular coagulation have all been associated with poorer clinical outcomes after the acquisition of Acinetobacter baumannii bloodstream infections [3,20].

Our study has some limitations we would like to comment on. Firstly, it is a retrospective observational study; therefore, subject to confounding. To minimize bias and missing values in our records, we used the CSF microorganism criterion to classify patients with or without CSFDI $[2,19,21]$. For the same reason, we did not categorize patients according to the presence of a respiratory/urinary tract infection, but rather focused on the role of bacteremia that we could accurately investigate in retrospect as blood is a sterile body fluid, and growth of pathogens cannot be attributed to colonization. Even though we aimed to find the role of prior bacteremia, we cannot exclude that, in some of the included cases, the bacteremia might have been ongoing at the time of the first positive CSF sample collection. Secondly, it is a single-center study, and our conclusions may not apply to all ICUs. Our results are, nonetheless, representative of a general ICU department that admits neurosurgical patients. Thirdly, the presence of a control group would, without doubt, have strengthened the result. In order to include the right patients for the control group, we should have identified the cases with central nervous system pathology that, for some reason, did not have an EVD or LD inserted but still had cerebrospinal fluid sampled by lumbar puncture during their stay in intensive care. However, such cases are exceptionally rare because lumbar puncture is usually contraindicated in severe intracranial pathology 
and, even if they exist, it is still extremely difficult to locate them retrospectively. Finally, the sample size of our study was not calculated in advance and may be too small for power analysis considerations. However, since the condition we studied is rare, our results may inform the design of future, multi-center studies for the investigation of similar hypotheses.

\section{Materials and Methods}

We designed a retrospective, single-center cohort study that took place at Evangelismos general hospital, Athens, Greece. The Evangelismos hospital ICU is a 25-bed general unit admitting both medical and surgical cases, including trauma. Patients with SARS-CoV-2 infection are admitted in a separate clinical area. The most common pathogens causing ICU-acquired infections in the department were, according to local surveillance data, Acinetobacter baumannii, Pseudomonas aeruginosa, and Klebsiella pneumoniae, comprising almost $75 \%$ of all positive cultures, while resistance to carbapenems was ranging from 40 to $95 \%$ for different pathogens during the study period. We reviewed the electronic hospital records of all ICU admissions from 1 January 2011 to 31 December 2020 to identify the eligible neurosurgical cases. The eligibility criteria were: presence of a CSF drain during ICU stay, time from drain insertion to ICU admission $<12 \mathrm{~h}$ or drain inserted after ICU admission, no previous diagnosis of CSF or any other infection prior to ICU admission, either hospital- or community-acquired, and length of ICU stay $>72 \mathrm{~h}$.

Data extracted from the electronic medical records included patient demographics; reason for ICU admission; date and time of ICU admission and discharge; ICU outcome; type of CSFD, i.e., EVD or LD; whether the drain used was impregnated with antibiotics or not; the date and time of CSF drain insertion and removal; and the number of CSF samples sent for analysis until the first positive CSF sample was documented or drain removal, whichever occurred first. We also recorded microbiology and laboratory data. For the CSF specimens, we recorded any microbial pathogens present, the white cell count, the white cell type, the red cell count, and the glucose and protein levels. For blood cultures, we recorded the cultured microorganisms, until the first positive CSF sample was obtained or the CSF drain was removed, whichever occurred first.

All culture specimens were ordered by the attending physicians when infection was suspected. Blood cultures were obtained via peripheral venous puncture using a standard sterile technique or from a new central venous catheter immediately after placement and prior to breaking the sterile field that was used for the catheterization. Blood cultures were performed using the BACTEC 9240 system (Becton-Dickinson Sparks, Baltimore, MD, USA). In the cases of coagulase-negative bacteremia, we applied criteria for detection of contamination [22]. We used the criterion of culture positivity [2,20] to identify CSF infections. In the cases where Staphylococcus epidermidis was the identified pathogen, we required two positive CSF cultures to classify it as infected [19]. Only blood cultures collected prior to the date of the first positive CSF sample or the drain being removed, whichever occurred first, were included in the analysis.

The study protocol was approved by the Ethics Committee of Evangelismos General Hospital (approval protocol number: 421/2018, with date of approval 13 November 2018). All investigations were conducted according to the principles expressed in the Declaration of Helsinki.

We used the R statistical software, version 3.6, for data visualization and statistical analysis. Continuous variables are reported as median (interquartile range). Count data are reported by frequencies. We used non-parametric statistical methods. Precisely, we used the Mann-Whitney $U$ test to compare the distributions of continuous variables between groups and the Fisher exact test to compare proportions between groups. Multivariate logistic regression was used to assess independent risk factors for CSF infection. The variables included in the model were selected according to previously described risk factors for CSFDI and included the type of CSFD, the length of stay, possible impregnation with antibiotics, and the number of CSF samples sent for analysis until the first positive CSF sample was documented or drain removal, whichever occurred first [2,11-14]. The 
Benjamini-Hochberg false discovery rate procedure [23] with tolerated false discovery rate $\alpha=0.05$ revealed a corrected $p$-value 0.03 to be significant for multiple comparisons.

\section{Conclusions}

In conclusion, our data support that bacteremia may be an important factor affecting the risk for CSFDI in critically ill patients, and we suggest that bacteremia be considered in future prospective and retrospective studies. In accordance with most recent reports, Acinetobacter baumannii was the commonest causative pathogen in our population. This represents a divergence from earlier reports, where Gram-positive bacteria of the skin flora were involved in the majority of CSFDI cases. Awareness of this change of epidemiologic profile is important for the prophylactic and empirical antibiotic coverage choices that need to take into account local surveillance data and patterns of resistance.

Author Contributions: Conceptualization, C.S.V., E.D., T.K. and A.K.; Data curation, M.N., G.B., C.L. and G.G.; Formal analysis, C.S.V., E.D. and T.K.; Investigation, G.B. and C.L.; Methodology, M.N.; Resources, A.K. and G.S.; Supervision, C.S.V., T.K. and G.S.; Validation, C.S.V., E.D., M.N., G.B., T.K., C.L., G.G., A.K. and G.S.; Visualization, E.D., A.K. and G.S.; Writing-original draft, C.S.V., G.B., T.K. and C.L.; Writing-review and editing, C.S.V., E.D., M.N., T.K., G.G., A.K. and G.S. All authors have read and agreed to the published version of the manuscript.

Funding: This research received no external funding.

Institutional Review Board Statement: The study was conducted in accordance with the Declaration of Helsinki and approved by the Ethics Committee of Evangelismos General Hospital (approval protocol number: 421/2018).

Informed Consent Statement: Patient consent was waived due to the retrospective, observational, and non-interventional nature of the study.

Data Availability Statement: The datasets generated during and/or analyzed during the current study are available from the corresponding author on reasonable request.

Conflicts of Interest: The authors declare no conflict of interest.

\section{References}

1. van de Beek, D.; Drake, J.M.; Tunkel, A.R. Nosocomial Bacterial Meningitis. N. Engl. J. Med. 2010, 362, 146-154. [CrossRef]

2. Tunkel, A.R.; Hasbun, R.; Bhimraj, A.; Byers, K.; Kaplan, S.L.; Scheld, W.M.; van de Beek, D.; Bleck, T.P.; Garton, H.J.L.; Zunt, J.R. 2017 Infectious Diseases Society of America's Clinical Practice Guidelines for Healthcare-Associated Ventriculitis and Meningitis. Clin. Infect. Dis. 2017, 64. [CrossRef]

3. Donlan, R.M.; Costerton, J.W. Biofilms: Survival Mechanisms of Clinically Relevant Microorganisms. Clin. Microbiol. Rev. 2002, 15, 167-193. [CrossRef]

4. Mounier, R.; Lobo, D.; Cook, F.; Martin, M.; Attias, A.; Ait-Mamar, B.; Gabriel, I.; Bekaert, O.; Bardon, J.; Nebbad, B.; et al. From the Skin to the Brain: Pathophysiology of Colonization and Infection of External Ventricular Drain, a Prospective Observational Study. PLOS ONE 2015, 10, e0142320. [CrossRef]

5. Ramírez, P.; Gordón, M.; Soriano, A.; Gil-Perotin, S.; Marti, V.; Gonzalez-Barbera, E.M.; Sanchez-Aguilar, M.T.; Simal, J.A.; Bonastre, J. Assessment of the in Vivo Formation of Biofilm on External Ventricular Drainages. Eur. J. Clin. Microbiol. Infect. Dis. 2013, 32, 1437-1443. [CrossRef]

6. Scheithauer, S.; Bürgel, U.; Ryang, Y.M.; Haase, G.; Schiefer, J.; Koch, S.; Häfner, H.; Lemmen, S. Prospective Surveillance of Drain Associated Meningitis/Ventriculitis in a Neurosurgery and Neurological Intensive Care Unit. J. Neurol. Neurosurg. Psychiatry 2009, 80, 1381-1385. [CrossRef]

7. Enoch, D.A.; Summers, C.; Brown, N.M.; Moore, L.; Gillham, M.I.; Burnstein, R.M.; Thaxter, R.; Enoch, L.M.; Matta, B.; Sule, O. Investigation and Management of an Outbreak of Multidrug-Carbapenem-Resistant Acinetobacter Baumannii in Cambridge, UK. J. Hosp. Infect. 2008, 70, 109-118. [CrossRef]

8. Citerio, G.; Signorini, L.; Bronco, A.; Vargiolu, A.; Rota, M.; Latronico, N. External Ventricular and Lumbar Drain Device Infections in Icu Patients: A Prospective Multicenter Italian Study. Crit. Care Med. 2015, 43, 1630-1637. [CrossRef]

9. Chu, S.M.; Hsu, J.F.; Lee, C.W.; Lien, R.; Huang, H.R.; Chiang, M.C.; Fu, R.H.; Tsai, M.H. Neurological Complications after Neonatal Bacteremia: The Clinical Characteristics, Risk Factors, and Outcomes. PLoS ONE 2014, 9, e105294. [CrossRef]

10. Akturk, H.; Sutcu, M.; Somer, A.; Aydin, D.; Cihan, R.; Ozdemir, A.; Coban, A.; Ince, Z.; Citak, A.; Salman, N. CarbapenemResistant Klebsiella Pneumoniae Colonization in Pediatric and Neonatal Intensive Care Units: Risk Factors for Progression to Infection. Braz. J. Infect. Dis. 2016, 20, 134-140. [CrossRef] 
11. Williamson, R.A.; Phillips-Bute, B.G.; McDonagh, D.L.; Gray, M.C.; Zomorodi, A.R.; Olson, D.W.M.; Britz, G.W.; Laskowitz, D.T.; James, M.L. Predictors of Extraventricular Drain-Associated Bacterial Ventriculitis. J. Crit. Care 2014, 29, 77-82. [CrossRef] [PubMed]

12. Murthy, S.B.; Moradiya, Y.; Shah, J.; Hanley, D.F.; Ziai, W.C. Incidence, Predictors, and Outcomes of Ventriculostomy-Associated Infections in Spontaneous Intracerebral Hemorrhage. Neurocritical Care 2016, 24, 389-396. [CrossRef] [PubMed]

13. Chen, C.H.; Chang, C.Y.; Lin, L.J.; Chen, W.L.; Chang, Y.J.; Wang, S.H.; Cheng, C.Y.; Yen, H.C. Risk Factors Associated with Postcraniotomy Meningitis: A Retrospective Study. Medicine 2016, 95, e4329. [CrossRef]

14. Camacho, E.F.; Boszczowski, I.; Freire, M.P.; Pinto, F.C.G.; Guimaraes, T.; Teixeira, M.J.; Costa, S.F. Impact of an Educational Intervention Implanted in a Neurological Intensive Care Unit on Rates of Infection Related to External Ventricular Drains. PLoS ONE 2013, 8, e50708. [CrossRef] [PubMed]

15. Williams, T.A.; Leslie, G.D.; Dobb, G.J.; Roberts, B.; van Heerden, P.V. Decrease in Proven Ventriculitis by Reducing the Frequency of Cerebrospinal Fluid Sampling from Extraventricular Drains: Clinical Article. J. Neurosurg. 2011, 115, 1040-1046. [CrossRef] [PubMed]

16. Thompson, D.R.; Vlachos, S.; Patel, S.; Innocent, S.; Tolias, C.; Barkas, K. Recurrent Sampling and Ventriculostomy-Associated Infections: A Case-Control Study. Acta Neurochir. 2018, 160, 1089-1096. [CrossRef]

17. Hader, W.J.; Steinbok, P. The Value of Routine Cultures of the Cerebrospinal Fluid in Patients with External Ventricular Drains. Neurosurgery 2000, 46, 1149-1155. [CrossRef]

18. Ramanan, M.; Lipman, J.; Shorr, A.; Shankar, A. A Meta-Analysis of Ventriculostomy-Associated Cerebrospinal Fluid Infections. BMC Infect. Dis. 2015, 14. [CrossRef]

19. Hussein, K.; Rabino, G.; Feder, O.; Eghbaryeh, H.; Zayyad, H.; Sviri, G.; Benenson, R.; Paul, M. Risk Factors for Meningitis in Neurosurgical Patients with Cerebrospinal Fluid Drains: Prospective Observational Cohort Study. Acta Neurochir. 2019, 161, 517-524. [CrossRef]

20. Wong, D.; Nielsen, T.B.; Bonomo, R.A.; Pantapalangkoor, P.; Luna, B.; Spellberg, B. Clinical and Pathophysiological Overview of Acinetobacter Infections: A Century of Challenges. Clin. Microbiol. Rev. 2017, 30, 409-447. [CrossRef]

21. Woo, P.Y.M.; Wong, H.T.; Pu, J.K.S.; Wong, W.K.; Wong, L.Y.W.; Lee, M.W.Y.; Yam, K.Y.; Lui, W.M.; Poon, W.S. Moving the Goalposts: A Comparison of Different Definitions for Primary External Ventricular Drain Infection and Its Risk Factors: A Multi-Center Study of 2575 Patients. J. Clin. Neurosci. 2017, 45, 67-72. [CrossRef] [PubMed]

22. Al Wohoush, I.; Rivera, J.; Cairo, J.; Hachem, R.; Raad, I. Comparing Clinical and Microbiological Methods for the Diagnosis of True Bacteraemia among Patients with Multiple Blood Cultures Positive for Coagulase-Negative Staphylococci. Clin. Microbiol. Infect. 2011, 17, 569-571. [CrossRef] [PubMed]

23. Benjamini, Y.; Drai, D.; Elmer, G.; Kafkafi, N.; Golani, I. Controlling the False Discovery Rate in Behavior Genetics Research. Behav. Brain Res. 2001, 125, 279-284. [CrossRef] 